\title{
EL ENCANTO DE 'FORMAR GENTE”. PASIONES E INFLUENCIAS DE LORANDI: UNA MIRADA DESDE LA SECCIÓN ETNOHISTORIA
}

\author{
THE ENCHANTMENT OF “FORM PEOPLE”. PASSIONS AND INFLUENCES \\ OF LORANDI: A LOOK FROM THE SECTION ETHNOHISTORY
}

\begin{abstract}
Julia Costilla*
En estas páginas recorreré algunas experiencias de mi vida académica en donde se podrán apreciar las influencias de Ana María Lorandi y su pasión por "formar gente". En función de mi trayectoria personal dentro del equipo creado y dirigido por Lorandi, abriré distintas puertas hacia la figura de esta investigadora. En primer lugar abordaré su papel como profesora del Departamento de Ciencias Antropológicas en la Universidad de Buenos Aires (UBA). A continuación, a partir de mis primeros vínculos con la Sección Etnohistoria del Instituto de Ciencias Antropológicas (UBA), iré desplegando otras facetas de Ana María: mentora de un Seminario de Investigación en Antropología Histórica, titular de cátedra en la materia "Sistemas Socioculturales de América II" y consejera de estudiantes y tesistas. Luego me detendré en el rol de Ana como investigadora, directora de proyectos y referente para becarios e investigadores. Finalmente atenderé a sus cualidades personales más destacadas, valoradas por todos aquellos que tuvieron el privilegio de conocerla.

Palabras claves: Ana María Lorandi, Sección Etnohistoria, Universidad de Buenos Aires, formación profesional, trayectoria académica.

In these pages I will follow some experiences of my academic life, where the influences of Ana Maria Lorandi and her passion for "training people" will be appreciate. According to my personal experience within the research team created and directed by Lorandi, I will open various doors to the figure of this researcher. First, I will address her role as a professor in the Anthropological Department at the University of Buenos Aires (UBA). Then, from my first bonds with the Section, I will display other facets of Ana Maria: mentor of a Research Seminar in Historical Anthropology, head teacher on the subject "Socio-cultural systems of America II" and counselor for students. From there, I'll continue with the influential role of Ana María as a researcher, as principalof collective projectsand as a reference for scholars and researchers. Finally I'll attend to their more prominent personal qualities, valued by all who had the privilege of meeting her.

Key words: Ana María Lorandi, Ethnohistory Section, University of Buenos Aires, professional training, academic trajectory.
\end{abstract}

\section{Introducción}

Definir el tema para una tesis doctoral es con frecuencia una tarea difícil. Al pensar en cómo Ana María marcó mi trayectoria profesional, el recuerdo más vivo que me asalta es el de estar en su casa conversando con ella acerca de mis variados y poco definidos intereses de investigación. Pero sobre todo retengo el momento en que con su lucidez logró orientarme en un tema y alentarme en un recorte espacial y temporal que parecía casi imposible de abordar. Es que ella tenía esa capacidad de estimular y generar confianza a la hora de tomar un desafío. Porque ella misma forjó su carrera profesional montada en un perseverante deseo de innovar y reinventar su trabajo, enfrentándose a los desafíos con entusiasmo, perspicacia y convicción.

Como se indica en un reciente artículo pertinente a su trayectoria, la labor profesional de Ana María se puede abordar desde distintos aspectos. Entre ellos, es posible destacar su inserción en redes transnacionales, la promoción de temas de investigación y la gestión institucional, sumadas a sus desarrollos conceptuales y metodológicos (Ramos y Chiappe 2016, cfr. Boixadós y Bunster 2016). Aunque excede a este trabajo abordar esos aspectos y revisar sus aportes a los campos (inter) disciplinares de la Etnohistoria y los estudios andinos, los hechos que relataré dan cuenta de sus acciones $\mathrm{y}$ contribuciones de una manera muy particular.

\footnotetext{
* Universidad de Buenos Aires, Facultad de Filosofía y Letras. Instituto de Ciencias Antropológicas. Buenos Aires, Argentina. Correo electrónico: juliacostilla@gmail.com
} 
Ana María siempre manifestó su pasión por "formar gente"; una pasión que supo transmitir a todos aquellos a quienes formó. Como parte del enorme grupo de afortunados que la conocieron y recibieron su formación, dedico estas páginas a recorrer algunas experiencias de mi vida académica en donde seguramente se podrá apreciar esa pasión de Ana María. ${ }^{1}$

En primer lugar abordaré su papel como profesora del Departamento de Ciencias Antropológicas en la Universidad de Buenos Aires (UBA). Luego, a partir de mis primeros vínculos con el equipo de investigación que la doctora Lorandi creó y dirigió durante 30 años -la Sección Etnohistoria del Instituto de Ciencias Antropológicas (UBA)- iré desplegando otras facetas de Ana María: mentora de un Seminario de Investigación en Antropología Histórica, titular de cátedra en la materia "Sistemas Socioculturales de América II" y consejera de estudiantes y tesistas. Desde allí llegaré al momento en el que consolidé mi vínculo con la Sección por medio de una beca doctoral y continuaré el camino hacia el influyente rol de Ana como investigadora, directora de proyectos y referente para los becarios e investigadores que nos iniciábamos en el mundo académico. Finalmente atenderé a sus cualidades personales más destacadas, valoradas por todos aquellos que tuvieron el privilegio de conocerla. Así, en función de mi trayectoria personal dentro del equipo de la Sección Etnohistoria, correspondiente a una generación de investigadores a quienes Ana María reconocía como sus "nietos", abriré distintas puertas hacia la figura de esta inolvidable investigadora.

\section{La Sección Etnohistoria y los legados de una pasión}

La primera imagen que rescato es la de la profesora Lorandi, titular de la materia -tan temida como amada- "Sistemas Socioculturales de América II". La materia suele cursarse en los últimos tramos de la carrera de Ciencias Antropológicas, dentro de la Facultad de Filosofía y Letras (FFyL) de la UBA. Conocer a Lorandi desde el banco de un aula de la Facultad, fue para mí escucharla con admiración y con un continuo interés por las historias y preguntas que traía a las clases. Desde mi experiencia, pero -me atrevo a decir-para la mayoría de quienes la tuvieron como profesora, ser estudiante de su materia significó abrir la mente a un mundo de información histórica y de reflexiones entre la antropología y la historia. En definitiva, conocer a Ana en ese contexto fue interesarme por una disciplina, a partir de escucharla y de acercarme a sus textos. Recuerdo el momento de estar leyendo su clásico trabajo con Mercedes del Río La Etnohistoria: etnogénesis y transformaciones sociales andinas (1992) y la sensación de estar encontrando en las palabras que leía, particularmente en la definición de Etnohistoria (p.10), una disciplina que me cautivó. Era una forma de hacer antropología que descubrí casi como una vocación latente, como el camino que quería seguir para mi futuro trabajo profesional.

En el programa de la materia del año 2003 también teníamos su libro Ni ley, ni rey, ni hombre virtuoso (2002), un texto que nos presentaba a personajes y hechos de la historia colonial sudamericana en una narrativa clara y atrapante. Más allá del gusto e interés por la materia, leer los capítulos de este libro resultaba al menos una tarea amena. Uno podía repasar sus páginas e imaginarse a Lorandi en el aula de clases teóricas contándonos de manera apasionada cada episodio de la historia e invitándonos a reflexionar acerca de ellos.

Al mismo tiempo, los docentes de la materia parecían estar impregnados por las mismas cualidades que su titular: entusiasmo, responsabilidad y dedicación. Todos ellos eran a la vez integrantes del equipo de investigación que dirigía Lorandi: la Sección Etnohistoria del Instituto de Ciencias Antropológicas. De esa manera, el contacto con los profesores nucleados en la cátedra de Lorandi podía conducir directamente a las puertas de las oficinas del $4^{\circ}$ piso, donde trabajaban $-\mathrm{y}$ trabajamos actualmente- los docentes e investigadores vinculados a la Etnohistoria. En general el primer acercamiento era para consultar algún libro o revista dentro de la amplia biblioteca de la Sección; pero también podía ser para despejar alguna duda con los profesores, siempre dispuestos a orientar a los estudiantes. Sin la materia "Socioculturales de América II" el camino que hoy transito tal vez no habría comenzado nunca.

A partir de ahí, mi vínculo con los integrantes de la Sección Etnohistoria continuó directamente con el Seminario de Investigación Anual, la instancia final donde los estudiantes orientados a la licenciatura definen su tema de tesis y diseñan su proyecto. Entre las opciones de seminarios anuales que ofrece el Departamento de Ciencias Antropológicas de la Facultad (FFyL, UBA), los estudiantes reconocen a este Seminario Anual por su orientación en "Antropología Histórica" y principalmente por 
el trabajo con fuentes documentales. Dictado por primera vez en 1998, con el nombre de Teoría y Metodología en Antropología Histórica, este seminario había estado inicialmente a cargo de la Dra. Lorandi junto con otros investigadores formados de la Sección. Mientras su nombre respondía a revisiones que por ese año se venían discutiendo en el equipo (respecto de teoría y metodología entre la Antropología y la Historia) ${ }^{2}$, el espacio en sí parecía responder a ese interés de la Dra. Lorandi por formar gente, interés que era por supuesto compartido con el resto de los docentes que lo integraban. Además de la materia "Socio II", donde la formación se dirigía a todos los estudiantes de Antropología, el Seminario profundizaba esa tarea para aquellos interesados en la labor investigativa.

Fue durante la cursada de ese Seminario, en el 2004, que comenzó formalmente mi recorrido académico dentro de la Sección Etnohistoria guiada y acompañada por quien terminó siendo director de mis sucesivas tesis, el Dr. Carlos Zanolli. Si bien Ana María no integraba físicamente el equipo de profesores que dictaban el curso en ese año (bajo la dirección de la Dra. Lidia Nacuzzi), su figura no dejaba de ser un referente. Entre los compañeros de ese curso, fuimos tres las estudiantes que continuamos como tesistas y becarias bajo la dirección de investigadores del equipo. Con las herramientas teórico-metodológicas recibidas de parte de los profesores de la cátedra, más la posibilidad de acceder a los libros, las fuentes documentales y el espacio de trabajo en las oficinas del $4^{\circ}$ piso de la Facultad, quienes iniciamos nuestro itinerario de investigación dentro del equipo de Etnohistoria -en distintas camadas anuales- hemos contado además con el plus de una experiencia acumulada en la producción de conocimientos y en la formación de recursos humanos. Y quien mejor representaba esa experiencia acumulada era Lorandi.

Inmediatamente después de cursar el Seminario Anual, es decir, durante los mismos años que dediqué a realizar la tesis de licenciatura, sumé un nuevo vínculo con el equipo de la Sección a partir de comenzar a trabajar como adscripta en la cátedra de "Socio II". Integrada en la comisión de clases prácticas a cargo de la entrañable profesora e investigadora María de Hoyos, me embarqué simultáneamente en las primeras experiencias entre docencia e investigación. De alguna manera, las actividades en la cátedra resultaron ser el complemento perfecto para la instancia individual de la tesis -no poco conflictiva y por momentos hasta asfixianteEl trabajo en las bibliotecas y los días dedicados a la escritura y la corrección de capítulos se iban alternando con los prácticos semanales de María y los encuentros frecuentes para discutir textos, preparar clases o corregir trabajos y exámenes. Por otra parte, asistir a las reuniones de cátedra implicaba conocer desde adentro la "cocina" de esa materia que tanto había admirado; participar junto con su equipo docente en el universo de discusiones y decisiones que enmarcan el dictado de las clases y la relación con los estudiantes. Pero en especial fue la instancia donde la "profesora Lorandi" comenzó lentamente a transformarse en Ana María, todavía como titular de la materia pero ya con una imagen más cercana y accesible, con quien los adscriptos podíamos dialogar desde un lugar distinto al que veníamos habituados desde nuestro rol de estudiantes.

La adscripción a "Socio II" y la tesis de licenciatura fueron las dos piedras fundacionales de mi formación como docente e investigadora, y al mismo tiempo fueron las dos instancias que consolidaron mi vínculo con la Sección Etnohistoria. En todo ese proceso, el papel de Ana María fue el de una guía académica ineludible. Aunque el acompañamiento y la orientación directa venían de Carlos Zanolli y de María de Hoyos, ella era de alguna manera el "Norte" de ambos espacios de trabajo: directora del equipo y titular de la cátedra.

Con la culminación casi simultánea de la adscripción a "Socio II" y de mi -prolongada-tesis de licenciatura, la mezcla de emociones propia de ese momento "liminar" se conjugó inmediatamente con la noticia de una beca doctoral de CONICET. El incentivo y la dedicación de quienes me acompañaron en esos primeros pasos académicos se materializaban al fin en una relación laboral que podía proyectarse a futuro. Las oficinas de la Sección Etnohistoria, que ya venían siendo para mí un espacio con el que empezaba a familiarizarme, se convirtieron definitivamente en mi "lugar de trabajo".

Ese cambio formal respecto de mi vínculo con el equipo de la Sección, había implicado también un cambio en cuanto a mi relación con Lorandi. Su imagen como titular de "Socio II" había derivado en la de Ana investigadora y directora de Sección. Empecé a conocerla en ese aspecto ya desde el momento que anticipé en la introducción: cuando -con bastante timidez-le pedí ayuda para definir el tema que presentaría para el doctorado. Ella lo aceptó 
con gusto y me ofreció inmediatamente recibirme en su casa para conversar concerniente al proyecto.

Allí le comenté hacia dónde venían orientandos mis intereses desde la finalización de mi licenciatura. Siempre me habían atraído las temáticas religiosas y simbólicas, y junto con mi director Carlos Zanolli estábamos pensando en un análisis comparativo y de larga duración entre cultos católicos sudamericanos. ${ }^{3}$ Mientras para la licenciatura me había enfocado en la simbología cristiana-indígena dentro del Noroeste argentino, la propuesta ahora apuntaba a ampliar el enfoque y la temporalidad con la inclusión de cultos correspondientes a distintos países y en una profundidad de casi cinco siglos. Por otra parte el problema que empezaba a delinear implicaba analizar distintos sectores de la sociedad americana entre la Colonia y la República: indígenas, hispano-criollos y afrodescendientes, en sus diferentes ocupaciones o posiciones de poder. Era por demás un proyecto ambicioso. Pero a pesar de ello, durante esa charla en su casa Ana me alentó a asumir el desafío y me ayudó a delinear los objetivos.

Recuerdo ese día con tanta gratitud que tal vez por ese motivo lo evoco con más fuerza al pensar en la influencia de Ana María en mi vida académica. Es frecuente que quienes la conocieron valoren su buena disposición a las consultas y afirmen que las puertas de su oficina -y de su casa- siempre estuvieron abiertas. Sin embargo, no dejo de destacar su gesto por lo que significó para mí, tanto en lo personal como en lo profesional, por haberme orientado y alentado en un problema de investigación con el que conseguí una beca y pude doctorarme. Este tipo de acciones son las que le valieron a Ana el reconocimiento casi unánime entre sus colegas por su generosidad.

Una vez iniciado el desarrollo de mi tesis doctoral, cada decisión importante pasaba primero por una charla con Ana para conocer su opinión y su consejo. Parte de su influencia ya la había recibido mediante la lectura de un artículo suyo escrito junto con Ana Edith Schaposchnik: Los milagros de la Virgen del Valle y la colonización de la ciudad de Catamarca (1990). En él se abordaba la colonización del Valle de Catamarca (dentro de la gobernación del Tucumán) y el sometimiento de la sociedad nativa en relación con los milagros de la Virgen y con la instalación del culto mariano en la zona. Uno de los puntos que más llamaron mi atención fue cómo por medio de un análisis de testimonios de la época, las autoras lograban identificar distintos significados para los milagros según intereses de los dos grandes sectores sociales que se cruzaron en el proceso de colonización: la población hispanocriolla y los nativos. Mientras para unos el milagro significaba dominación y sometimiento, para los otros implicaba la posibilidad de integrarse como cristianos en la sociedad colonial, aunque fuera desde un lugar subordinado.

La lectura de ese trabajo de Lorandi y Schaposhnik tuvo para mí un valor clave, por dos motivos. En principio porque fue gracias a él que empecé a reflexionar acerca de los milagros como símbolos religiosos -polisémicos y apropiados desde diferentes sectores sociales- y a prever la posibilidad de analizar en la tesis los usos sociopolíticos del milagro cristiano. Pero también porque fue un ejemplo claro de cómo podía articular mis intereses atinente a lo religioso y lo simbólico con un problema de investigación que entendía como típicamente etnohistórico.

Tomando en cuenta los temas tradicionalmente abordados por los miembros de la Sección, orientados en general al estudio de las sociedades indígenas en el período colonial -particularmente en el área andina-, mi propuesta de tesis se alejaba en cierta medida de esa línea de trabajos. Tomaba cultos de distintas zonas geográficas (el altiplano boliviano, la costa peruana y la región pampeana argentina), consideraba distintos actores sociales y abarcaba diferentes períodos históricos. Sin embargo, ya desde hacía unos años los problemas que se venían planteando en el equipo habían ampliado el enfoque social, regional y temporal. ${ }^{4}$ Así, comenzaron a contemplarse distintos sectores de la sociedad, distintas áreas geográficas y diferentes temporalidades, incluso llegando hasta el presente. En ese sentido, mi lugar como tesista/becaria puede entenderse como parte de una generación de investigadores que corporizaron un cierto giro temático. Giro que, por otra parte, no excluyó a los investigadores previos: la misma Ana María, por ejemplo, venía ya enfocando su mirada en la sociedad hispano-criolla.

Una instancia donde se podía evidenciar esa amplitud temática, y que fue muy relevante respecto de mi integración en la Sección Etnohistoria, fue la de los proyectos de investigación UBACyT y PICT $^{5}$. Desde mi llegada al equipo como tesista de licenciatura había comenzado a participar en actividades colectivas con otros miembros de la Sección y sin duda las más importantes fueron estos proyectos generales. En ellos Ana articulaba, con 
eficacia, problemáticas de las más variadas para elaborar planes colectivos e inclusivos. Sumarme a esos proyectos, ya históricos para el equipo, significó para mí conocer una nueva faceta de Ana desde la gestión y la planificación académica. Más allá de la inestimable posibilidad de contar con recursos para viajes de campo y congresos, descubrir el trasfondo de la preparación de los planes de trabajo, de la administración de los subsidios y de la ejecución de las actividades fue una instancia más de aprendizaje a partir del ejemplo de Ana como hábil directora de ambos proyectos.

Es decir que durante todo el proceso de mi tesis doctoral, el vínculo que tenía con Ana María se fue completando a partir de sus distintas facetas como investigadora: directora de la Sección, responsable de los proyectos colectivos, etnohistoriadora reconocida y consejera general para todos aquellos que iniciábamos nuestra formación profesional. Estas dos últimas facetas en particular son las que más destaco respecto de su influencia sobre mi trabajo. En primer lugar por las dos instancias que describí anteriormente: la lectura de su texto con Schaposhnik y la charla en su casa para definir mi tema. Luego por las consultas que solía hacerle cada vez que planeaba un viaje de campo o que decidíamos con Carlos Zanolli dar algún "volantazo" en cuanto al tema de investigación. Para los trabajos de campo y archivo Ana siempre tenía algún contacto académico clave en las ciudades que visitaba y para las decisiones importantes siempre supo aconsejarnos y guiarnos en las mejores opciones.

Al mismo tiempo, en esos años de formación, fueron llegando las ocasiones de compartir con Ana congresos, seminarios, viajes, reuniones y eventos académicos variados, como así también festejos con el equipo para diversas circunstancias (varias de estas la tenían como protagonista). Ya fueran encuentros académicos o sociales, ella siempre se mostraba igual: cordial, llana, franca, atenta, alegre y con una picardía característica. Gracias a esas oportunidades pude conocer un poco más a Ana en lo personal, acerca de sus historias, sus seres queridos y sus siempre divertidas anécdotas; sabía disfrutar de las comidas y bebidas compartidas, pero sobre todo sabía escuchar y conversar.

Todo ese recorrido que me llevó al lugar que ocupo hoy como integrante de la Sección Etnohistoria, estuvo marcado de una u otra manera por la influencia de Lorandi. Desde el día en que los profesores de "Socio II" se presentaron frente a los estudiantes del curso 2003, comencé a sentir una mezcla de interés y admiración por ese equipo docente que parecía tan sólido y armónico. La misma sensación tuve cuando conocí a los investigadores que trabajaban en las oficinas de Etnohistoria. Poder integrarme en ese espacio académico dirigido por Lorandi fue una gran satisfacción y a la vez un desafío por saber que debía estar a la altura de esa imagen -idealizadaque respecto de él tenía. Con el tiempo fui forjando vínculos y amistades, y por supuesto aprendiendo también de los conflictos que, como en cualquier ámbito de trabajo, se despertaron en algunas oportunidades. Estos últimos, sin embargo, no llegaban a opacar o a obstaculizar la producción académica y el profesionalismo del equipo. En general, por encima de esas eventuales tensiones, ha prevalecido el compañerismo, el espíritu de trabajo colectivo y la responsabilidad con las tareas de investigación, docencia, gestión y formación de recursos humanos.

Como señalamos con algunos miembros del equipo, algo que siempre destacó Ana es una forma particular de investigar y una manera "rigurosa" de llevar adelante el trabajo profesional. De distintas maneras y en distintos espacios ha promovido la valoración, el ejercicio y la transmisión de cualidades como seriedad, solidez, compromiso y tenacidad, entre otras (Zanolli et al. 2010: 102-103). Más allá de las aptitudes personales de cada uno de los investigadores y docentes que han sido parte de la Sección Etnohistoria y que se han formado con Ana María, esa manera de investigar es valorada como un rasgo que caracteriza a la Sección, tanto para sus miembros como para los colegas de otras secciones e institutos de la Facultad. Incluso por fuera de la UBA, le ha dado al equipo un notable reconocimiento entre colegas de otras universidades e institutos, en Argentina y en distintos países vinculados a la Etnohistoria y los estudios andinos. ${ }^{6}$ Debido a la trayectoria de Ana como creadora, mentora y directora del equipo, cuesta pensar a esas cualidades al margen de su influencia.

Este año, cuando decidimos entre los miembros de la Sección Etnohistoria honrar su memoria mediante una placa con su nombre, una opción fue bautizar la oficina que ella había ocupado por tres décadas como directora de Sección. Pero en seguida surgió la propuesta de hacerlo en la oficina donde ella solía reunir al equipo, donde compartimos charlas casuales, discusiones, seminarios, reuniones generales y brindis de fin de año. Mientras la primera opción la representaba a ella y a su lugar pionero 
como creadora de la Sección, de alguna manera esta segunda opción simbolizaba más su vínculo con el equipo y su lugar central como nexo entre todos.

En una entrevista que le realizamos en marzo del 2009, Ana María nos expresaba su pasión con estas palabras: "me encanta formar gente, lo hice con tanta ganas...". Ocho años después, este humilde homenaje intenta subrayar esa faceta de Ana María que siempre se mantuvo viva, que la convirtió en una maestra inagotable, y que hoy recordamos con profundo agradecimiento.

\section{Referencias Citadas}

Boixadós, R. y Bunster, C. (Eds.).

2016 Disciplinas sin fronteras. Homenaje a Ana María Lorandi. Editorial de la Facultad de Filosofía y Letras (UBA), Buenos Aires.

Costilla, J.

2015 "Itinerarios antropológicos para una Etnohistoria comparada de la religión: cultos católicos americanos en la larga duración”. Diálogo Andino 49: 299-309.

Chiappe, C. M.

2015 "Imaginarios en pugna: la politización del campo de los estudios andinos en el período fundacional de la etnohistoria andina chilena". Diálogo Andino 49: 287-297.

Lorandi, A. M. y M. Del Río

1992 La Etnohistoria: etnogénesis y transformaciones sociales andinas. CEAL, Buenos Aires.

Lorandi, A. M. y A. E. Schaposchnik

1990 "Los milagros de la Virgen del Valle y la colonización de la ciudad de Catamarca". Journal de la Société des Américanistes 76: 177-198. París.
Lorandi, A. M.

2009 Ni ley, ni rey, ni hombre virtuoso. Guerra y sociedad en el virreinato del Perú. Siglos XVI y XVII. GEDISA, Buenos Aires.

Ramos, A. y C. M. Chiappe

2016 "Ana María Lorandi y el tren de la Etnohistoria". Runa 37 (2): 97-113.

Zanolli, C., A. Ramos, D. Estruch y J. Costilla

2015 "La Etnohistoria en la Universidad de Buenos Aires: un recorrido por 30 años de investigaciones". Diálogo Andino 49.

Zanolli, C., A. Ramos, D. Estruch y J. Costilla 2010 Historia, representaciones y prácticas de la Etnohistoria. Una aproximación antropológica a un campo de confluencia disciplinar. Antropofagia, Buenos Aires.

Zanolli C., J. Costilla, D. Estruch y A. Ramos (Comps.)

2013 Los estudios andinos hoy. Práctica intelectual y estrategias de investigación. Prohistoria, Rosario.

\section{Notas}

1 Este artículo se propone enteramente como un homenaje a la labor de la Dra. Lorandi en la Sección Etnohistoria del Instituto de Ciencias Antropológicas, Universidad de Buenos Aires. In memoriam.

2 Véase Zanolli et al. 2010

Véase Costilla 2015.

Cfr. Zanolli et al. 2010, 2015.
5 Proyectos acreditados y financiados respectivamente por la Universidad de Buenos Aires y por la Agencia Nacional de Promoción Científica y Tecnológica, dependiente del Ministerio de Ciencia, Tecnología e Innovación Productiva de la Nación.

$6 \quad$ Véase Zanolli et al. 2013. 